\title{
Maxillary dentinogenic ghost cell tumour
}

\author{
TC Biggs, SM Hayes, PG Harries, RJ Salib
}

\section{University Hospital Southampton NHS Foundation Trust, UK}

\section{ABSTRACT}

Dentinogenic ghost cell tumours are extremely rare, and pose significant diagnostic and therapeutic challenges as this case clearly demonstrates. An awareness of different clinical presentations and distinct histopathological features is important in establishing an early definitive diagnosis and instituting appropriate management. Furthermore, there is little precedent in the literature to guide management in such a case, and we therefore consider this report to be noteworthy and instructive in this respect.

\section{KEYWORDS}

Dentinogenic - Odontogenic - Ghost cell - Tumour - Diagnosis - Pathology

Accepted 19 November 2012; published online 05 April 2013

\section{CORRESPONDENCE TO}

Timothy Biggs, ENT Department, University Hospital Southampton NHS Foundation Trust, Tremona Road, Southampton SO16 6YD, UK $\mathrm{T}:$ +44 (0)7734 816 487; F: +44 (0)23 8079 4868; E: tim_biggs@ymail.com

Dentinogenic ghost cell tumours are extremely rare, posing significant diagnostic and therapeutic challenges. ${ }^{1}$ In 2005 the World Health Organization classified them into three groups: calcifying cystic odontogenic tumours (CCOTs), described as benign cystic neoplasms of odontogenic origin; dentinogenic ghost cell tumours (DGCTs), described as locally invasive neoplasms characterised by ghost cell formation; and ghost cell odontogenic carcinomas (GCOCs), described as malignant and aggressive neoplasms containing groups of ghost epithelial cells, with metastatic potential. ${ }^{1}$ Our case report describes a recurrent maxillary DGCT in a 27-year-old man that presented initially as a painful cheek swelling with associated symptoms of maxillary sinus outflow obstruction.

\section{Case history}

A 27-year-old Afro-Caribbean man presented with a rightsided cheek swelling steadily increasing in size over a 3-week period, associated with right-sided severe cheek pain and nasal obstruction. There was no history of blood stained nasal discharge. He was a sickle cell carrier but otherwise fit and well. He was a non-smoker and took no regular medications.

On examination, there was a right-sided palatal bulge, with a $5 \mathrm{~cm} \times 4 \mathrm{~cm}$ firm, painful swelling overlying the right maxilla and right nasal alar. Anterior rhinoscopy revealed a right-sided medially displaced inferior turbinate with an unusual looking red swelling extending from the medial wall of the maxillary sinus. Detailed endoscopic examination was limited owing to poor access and severe discomfort. There was no palpable lymphadenopathy in the neck. Ultrasonography suggested a cystic lesion deep to the zygoma, with a possible infective aetiology, and antibiotics were therefore commenced. Computed tomography (CT) confirmed the presence of a large, rounded, cystic-looking structure arising from the right maxillary alveolus, extending into the maxillary antrum. Remodelling of the bony lateral and medial walls had occurred, and the overall appearances at that stage were suggestive of a calcifying odontogenic cyst (COC) (Fig 1).

It was presumed that the COC had developed as a result of a supernumerary tooth fragment. Therefore, surgical exploration, biopsy and enucleation were carried out via a Caldwell-Luc approach, revealing a thick lined cyst adherent to the walls of the maxillary sinus. Histopathological examination revealed a fibrous walled cyst lined with squamous epithelium and predominant areas of ghost cell keratinisation, appearances consistent with those of a benign COC.

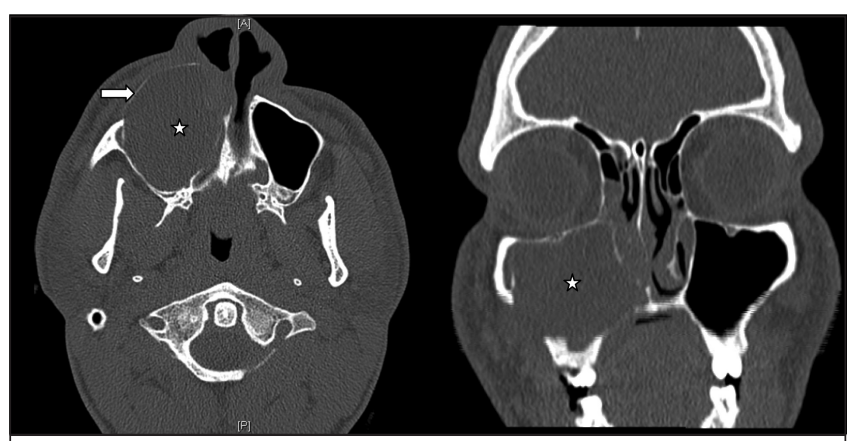

Figure 1 Computed tomography (axial and coronal planes), showing a large rounded mass with cystic features in the right maxillary sinus (white star). The mass has a thin bony cortex (white arrow) with evidence of remodelling of the bony lateral and medial walls of the maxillary sinus. 


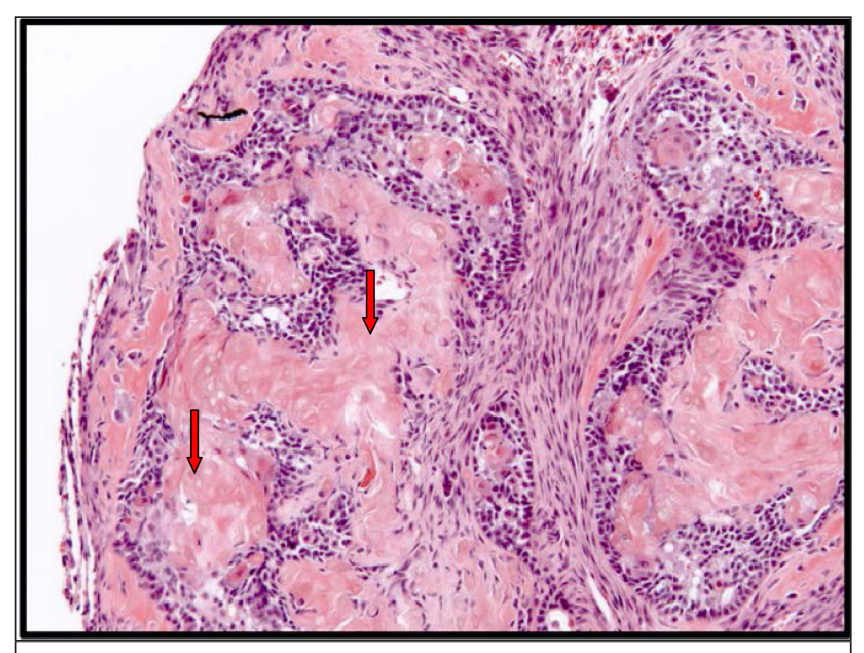

Figure 2 Haematoxylin and eosin staining of odontogenic epithelium showing prominent ghost cell formation (red arrows)

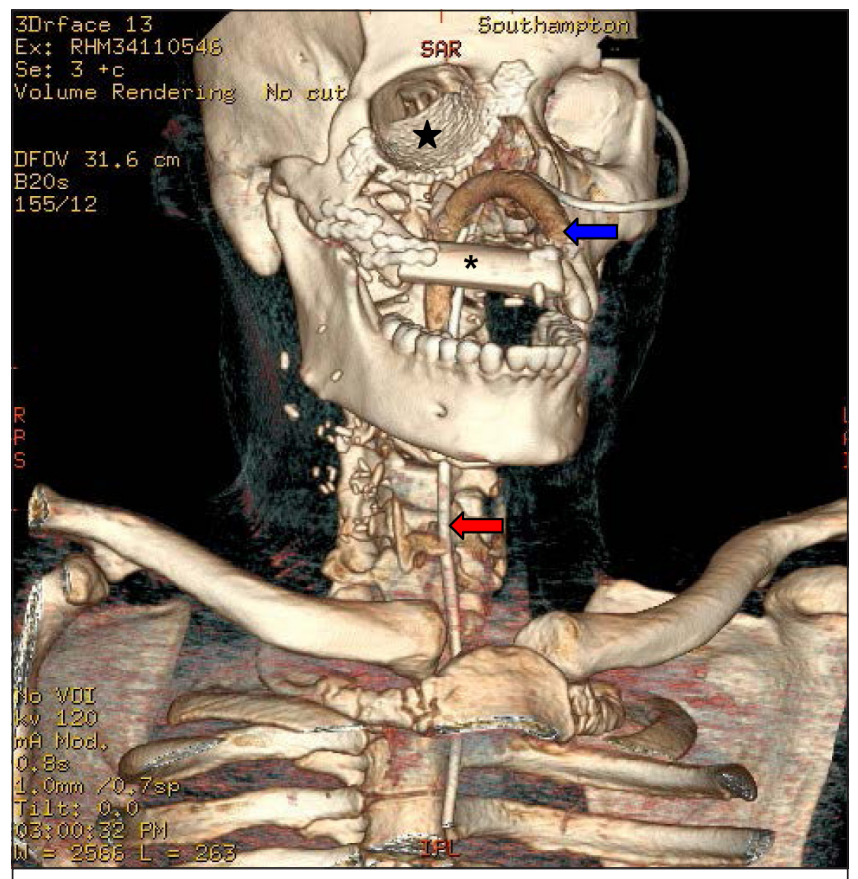

Figure 3 Three-dimensional computed tomography highlighting the fibula free graft (black asterisk) and orbital reconstruction (black star) following final tumour resection and maxillectomy. A nasopharyngeal airway (blue arrow) and nasogastric tube (red arrow) are also in situ.

Over the following six weeks, the patient presented as an emergency on two separate occasions, with persistent rightsided facial swelling and a unilateral right-sided maxillary sinusitis. It was felt that the worsening symptoms were due to a right ostiomeatal complex obstruction secondary to distortion of the lateral nasal wall. Endoscopic examination at that time revealed a bulging mass occluding the nasal airway, with possible involvement of the soft tissue of the floor of the nasal cavity and cheek. He therefore underwent a right transnasal endoscopic medial maxillectomy, revealing a cystic structure filled with keratinous debris. The histological appearances were that of a solid lesion comprising odontogenic epithelium with prominent ghost cell formation. The features were those of a DGCT (Fig 2).

Within three months postoperatively, the patient presented once again with increased pain and a firm swelling in the region of the right maxilla. Repeat CT revealed that the tumour had recurred and it was decided following discussion at the multidisciplinary head and neck meeting to undertake a right-sided hemimaxillectomy with maxillary reconstruction using a free fibula flap and titanium mesh (Fig 3).

Repeated CT at follow-up appointments has shown no evidence of any tumour recurrence and the patient remains asymptomatic at 12 months. He will be monitored closely in the multidisciplinary head and neck oncology clinic, particularly as recurrence is a common feature of these tumours.

\section{Discussion}

Ghost cell odontogenic tumours are very rare with only a handful of reports in the literature. ${ }^{1}$ They are characterised by the formation of ghost cells, which are eosinophilic epithelial cells containing no nucleus. CCOTs are the most common of all three types and present normally as a slowgrowing painless cystic structure. Radiologically, they appear as a well defined radiolucent mass and rarely recur following surgical excision. In contrast, DGCTs are more aggressive and much more likely to recur. GCOTs are frankly malignant lesions often occurring as a result of malignant transformation in a CCOT or DGCT. The tumour described in this case was that of a maxillary DGCT.

DGCTs are reported as occurring peripherally or centrally. Peripheral tumours typically arise in the extraosseous gingival or alveolar mucosa. ${ }^{2}$ These lesions behave less aggressively and can normally be managed by local excision alone. Central tumours usually arise in the maxilla or mandible and growth tends to be infiltrative in nature. ${ }^{3-5}$ They therefore require more radical surgery, frequently with the addition of adjuvant radiotherapy.

Rarely, tumours can present simultaneously in multiple sites. ${ }^{3}$ The age of presentation is variable (between 13 and 92 years of age), typically occurring in Asian individuals, with a higher incidence seen in males (4:1 male-to-female ratio). ${ }^{1}$ The presentation of a DGCT is normally with a painful swelling in the area of the jaw or maxilla. ${ }^{5}$ In this case report, the patient presented with a painful swelling, nasal obstruction and maxillary sinus outflow obstruction. This presentation has not been reported previously. Radiographically, these tumours are characterised by ill-defined radiolucency and bone resorption. ${ }^{5}$

The diagnosis of DGCT is a histopathological one, revealing a well-defined basal layer of columnar cells with masses of ghost epithelial cells with or without calcification. ${ }^{1,5}$ Surgical excision with wide histological margins (at least $0.5 \mathrm{~cm}$ ) is recommended as the primary mode of management, with postoperative radiotherapy in selected cases, ${ }^{5}$ and multidisciplinary discussion prior to and follow- 
ing treatment. Local recurrence can occur (5-17 years following presentation) in up to $16-70 \%$ of cases and distant metastasis has also been reported. ${ }^{5}$ Consequently, the management of these cases should mirror that of any malignant tumour of the head and neck, with baseline postoperative magnetic resonance imaging, interval imaging and longterm surveillance. ${ }^{1}$

\section{References}

1. Ledesma-Montes C, Gorlin RJ, Shear M et al. International collaborative study on ghost cell odontogenic tumours: calcifying cystic odontogenic tumour, dentinogenic ghost cell tumour and ghost cell odontogenic carcinoma. J Oral Pathol Med 2008; 37: 302-308.

2. Kasahara $\mathrm{K}$, lizuka $\mathrm{T}$, Kobayashi I et al. A recurrent case of odontogenic ghost cell tumour of the mandible. Int J Oral Maxillofac Surg 2002; 31: 684-687.

3. Basile JR, Klene C, Lin YL. Calcifying odontogenic cyst with odontogenic keratocyst: a case report and review of the literature. Oral Surg Oral Med Oral Pathol Oral Radiol Endod 2010; 109: e40-e45.

4. Sun G, Huang X, Hu Q et al. The diagnosis and treatment of dentinogenic ghost cell tumor. Int J Oral Maxillofac Surg 2009; 38: 1,179-1,183.

5. Cheng Y, Long X, Li X et al. Clinical and radiological features of odontogenic ghost cell carcinoma: review of the literature and report of four new cases. Dentomaxillofac Radiol 2004; 33: 152-157. 